\title{
CORRECTION
}

\section{Correction to: The Association Between Phosphorylated Neurofilament Heavy Chain (pNF-H) and Small Fiber Neuropathy (SFN) in Patients with Impaired Glucose Tolerance}

Yu-peng Li (1) · Zhong-qing Yan · Li-ping Han · Ai-li Yin •

Jin-yong Xu • Ya-ran Zhai · Sai Hao $\cdot$ Lin Zhang $\cdot$ Yun Xie

Published online: December 21, 2019

(C) The Author(s) 2019

Correction to:

Diabetes Ther

https://doi.org/10.1007/s13300-019-00716-w

In the original article, there was some error in Table 2 . The correct Table 2 is given below.

The original article can be found online at https://doi. org/10.1007/s13300-019-00716-w.

Y. Li (ه) - Z. Yan · L. Han · A. Yin · J. Xu · Y. Zhai · S. Hao · L. Zhang · Y. Xie $(\bowtie)$

Tianjin Medical University Metabolic Diseases

Hospital \& Tianjin Medical University Chu Hsien-I Memorial Hospital, Tianjin, China e-mail: dr_liyupeng@126.comY. Xie e-mail: xieyuntj@126.com

Y. Li · Z. Yan - L. Han - A. Yin · J. Xu · Y. Zhai .

S. Hao $\cdot$ L. Zhang $\cdot$ Y. Xie

Tianjin Institute of Endocrinology, Tianjin, China

Y. Li · Z. Yan · L. Han · A. Yin · J. Xu · Y. Zhai ·

S. Hao $\cdot$ L. Zhang $\cdot$ Y. Xie

NHC Key Laboratory of Hormones and

Development (Tianjin Medical University), Tianjin

Key Laboratory of Metabolic Diseases, Tianjin,

China 
Table 2 Basic demographical and clinical parameters among three groups

\begin{tabular}{|c|c|c|c|c|}
\hline & \multirow[t]{2}{*}{$\mathrm{CON}(N=33)$} & \multicolumn{2}{|c|}{ IGT $(N=61)$ (exclude 17 cases large fiber neuropathy) } & \multirow[t]{2}{*}{$p$ value } \\
\hline & & Non-SFN control & SFN & \\
\hline$N$ & 33 & 20 & 24 & \\
\hline $\mathrm{M} / \mathrm{F}$ & $10 / 23$ & $9 / 11$ & $11 / 23$ & 0.521 \\
\hline Age (years) & $50.5 \pm 8.7$ & $53.6 \pm 7.8$ & $57.7 \pm 8.8^{*}$ & 0.010 \\
\hline BMI $\left(\mathrm{kg} / \mathrm{m}^{2}\right)$ & $23.9 \pm 2.7$ & $25.22 \pm 2.53$ & $25.13 \pm 3.49$ & 0.175 \\
\hline WHR & $0.87 \pm 0.07$ & $0.9 \pm 0.07$ & $0.9 \pm 0.08$ & 0.125 \\
\hline HbAlc $(\%)$ & $5.6 \pm 0.3$ & $5.98 \pm 0.39^{*}$ & $5.93 \pm 0.39^{*}$ & 0.002 \\
\hline $\mathrm{FPG}(\mathrm{mmol} / \mathrm{l})$ & $5.02 \pm 0.32$ & $5.92 \pm 0.53^{*}$ & $5.89 \pm 0.53^{*}$ & 0.000 \\
\hline $2 \mathrm{hPG}(\mathrm{mmol} / \mathrm{l})$ & $6.02 \pm 1.11$ & $8.87 \pm 0.75^{*}$ & $9.52 \pm 0.92^{*}, \#$ & 0.000 \\
\hline $\mathrm{TG}(\mathrm{mmol} / \mathrm{l})$ & $1.52 \pm 0.57$ & $1.62 \pm 0.45$ & $1.60 \pm 0.99$ & 0.881 \\
\hline $\mathrm{TC}(\mathrm{mmol} / \mathrm{l})$ & $5.59 \pm 0.73$ & $5.15 \pm 1.01$ & $5.00 \pm 0.99$ & 0.070 \\
\hline HDL-c $(\mathrm{mmol} / \mathrm{l})$ & $1.58 \pm 0.45$ & $1.51 \pm 0.35$ & $1.39 \pm 0.31$ & 0.230 \\
\hline LDL-c (mmol/l) & $3.56 \pm 0.66$ & $3.11 \pm 0.88$ & $3.10 \pm 0.96$ & 0.134 \\
\hline $\mathrm{Cr}(\mathrm{mmol} / \mathrm{l})$ & $71.47 \pm 15.25$ & $69.25 \pm 10.90$ & $70.24 \pm 17.3$ & 0.883 \\
\hline BUN (mmol/l) & $5.04 \pm 1.49$ & $5.56 \pm 0.58$ & $5.68 \pm 1.13$ & 0.132 \\
\hline $\operatorname{ACR}(\mathrm{mg} / \mathrm{mmol})$ & $0.76(0.49,1.50)$ & $1.12 \pm 0.68$ & $0.71(0.57,1.88)$ & 0.891 \\
\hline
\end{tabular}

Continuous, normally distributed variables are expressed as means \pm SD and non-normally distributed variables are expressed as medians (25\% and $75 \%$ quartiles)

$M$ male, $F$ female, $B M I$ body mass index, $W H R$ waist to hip ratio, $H b A 1 c$ hemoglobin Alc, $F P G$ fasting plasma glucose, $2 h P G 2 \mathrm{~h}$ postprandial plasma glucose, $T G$ triglycerides, $T C$ total cholesterol, $H D L-c$ HDL-cholesterol, $L D L-c$ LDLcholesterol, $C r$ creatinine, $B U N$ blood urea nitrogen, $A C R$ urinary albumin to urinary creatinine ratio ${ }^{*} p<0.05$ compared to CTRL, ${ }^{*} p<0.05$ compared to IGT, based on one-way ANOVA with SNK analysis, Kruskal-Wallis $\mathrm{H}$ test or $\mathrm{v}$ test, as appropriate

\section{OPEN ACCESS}

This article is distributed under the terms of the Creative Commons Attribution-NonCommercial 4.0 International License (http:// creativecommons.org/licenses/by-nc/4.0/), which permits any noncommercial use, distribution, and reproduction in any medium, provided you give appropriate credit to the original author(s) and the source, provide a link to the Creative Commons license, and indicate if changes were made. 